\title{
Thermodynamics and Viscometric Studies of Monochloroacetic Acid in Water-Isopropanol Solvent Mixtures at 298, 303 and $308 \mathrm{~K}$
}

\author{
NIKAM NEENA PANDIT \\ Department of Chemistry, Savitri Bai Phule Pune University, \\ K.S.K.W.A.S.C. College, CIDCO, Nashik-422008, India \\ neenanikamchem@gmail.com
}

Received 14 October 2016 / Accepted 7 November 2016

\begin{abstract}
Ultrasonic velocity, density and viscosity of monochloroacetic acid (MCAA) solutions in $10,20,30,40,50,60,70,80,90$ and $100 \mathrm{wt}$ \% isopropanol-water have been determined at 298.15,303.15 and 308.15 K. Viscosity and density data of these solutions were analysed with Johndoles and Masson's equation. The viscosity coefficient A and B, the limiting apparent molar Volume $\left(\Phi^{0}{ }_{\mathrm{v}}\right)$ and experimental slop $\left(\mathrm{S}_{\mathrm{K}}\right)$, ultrasonic velocity $(\mathrm{U})$ and derived parameters namely, adiabatic compressibility $\left(\beta_{\mathrm{ad}}\right)$, apparent molar compressibility $\left(\Phi_{\mathrm{k}}\right)$, intermolecular free length $\left(\mathrm{L}_{\mathrm{f}}\right)$, specific acoustic impedance $(Z)$ and salvation number $\left(S_{n}\right)$ throw light on solute-solvent and solutesolute interactions. Consequence of temperature disparity on these interactions is discussed.
\end{abstract}

Keywords: Density, Viscosity, Ultrasonic Velocity, Adiabatic compressibility, Apparent molar compressibility, Specific acoustic impedance, Salvation number

\section{Introduction}

Precise thermodynamics and transport properties on working fluid are very often obligatory. The density and ultrasonic velocity data are significant basic properties to investigation mass transfers and compressibility of scientifically prospective fluids. Transport properties of electrolytes in aqueous, non-aqueous and mixed solvents are of concern in assorted technologies like high energy density batteries, electro deposition and in electr-oganic synthesis ${ }^{1}$. Viscosity, density and ultrasonic velocity measurements of electrolyte solutions are admirable apparatus to perceive solute-solvent and solute-solute interaction. These interactions in the case of electrolyte in water- $n$-alkanols mixtures have been deliberate by many workers, but such study in water-isoalcohol solvent mixtures are inadequate. It has been reported ${ }^{2}$ that isopropyl alcohol differs significantly from $n$-propyl alcohol, the way it undergoes protonation by solvated proton. Therefore viscosity, density and ultrasonic behaviours of the electrolyte are expected to demonstrate entirely different performance in water - isopropanol mixtures. Hence in order to inspect solute- solvent and solute- solute 
interaction in MCAA solutions in water - isopropanol, the present investigation of viscosity, density and ultrasonic velocity measurements of MCAA solutions in aqueous isopropanol of different dielectric constants is undertaken. In accumulation, an effort has been made to study the consequence of adding of MCAA on intermolecular interactions leading to hydrogen bond formation amid of water and isopropanol molecules ${ }^{3}$.

\section{Experimental}

MCAA was purified as reported previously ${ }^{4}$. Isopropanol was dried by refluxing with merged calcium oxide for 8-10 hours and ultimately distilled by means of a fractionating column. Triple distilled water was used to merge (by weight) with purified isopropanal to give mixtures of different dielectric constant ${ }^{5}$. Solutions of different molarities of MCAA were arranged again by dissolving acid in the solvent mixtures. Viscosity, density and ultrasonic velocity of the solution were calculated in thermostated water bath having thermal stability of $\pm 0.01{ }^{\circ} \mathrm{C}$, with $\mathrm{M}$-82 interferometer having frequency of $1.5 \mathrm{MH}_{\mathrm{z}}$, suspended level Ubbelohde viscometer and bicapillary pycnometer with an accuracy of $0.03 \%, \pm 0.03$ $\mathrm{C}_{\mathrm{p}} \pm 0.001 \mathrm{gm} / \mathrm{mL}$ respectively. The efflux times were calculated with stopwatch correct to 0.01 second.

\section{Results and Discussion}

The apparent molar volumes $(\Phi v)$ of MCAA in water - isopropanol solvent mixtures at all temperatures were calculated from there density data, obeyed Masson equation.

$$
\Phi v=\Phi_{v}{ }^{0}+S v \sqrt{C}
$$

The intercepts $\left(\Phi_{\mathrm{v}}\right)$ and slopes $\left(\mathrm{S}_{\mathrm{v}}\right)$ of linear plots of $\Phi \mathrm{v}$ versus $\sqrt{C}$ for all temperature are recorded in Table 1.

Table 1. $\Phi_{\mathrm{V}}{ }^{0}$ and $S_{\mathrm{V}}$ at all compositions and at all temperatures

\begin{tabular}{|c|c|c|c|c|c|c|}
\hline Temp. ${ }^{\circ} \mathrm{C}$ & $\begin{array}{c}\Phi_{\mathrm{v}}{ }^{0} \\
\mathrm{~cm}^{3} \mathrm{~mol}^{-1}\end{array}$ & $\begin{array}{c}\mathrm{SV} \mathrm{cm} \\
\mathrm{L}^{1 / 2} \mathrm{~mol}^{-3 / 2}\end{array}$ & $\begin{array}{c}\Phi_{\mathrm{v}}{ }^{0} \\
\mathrm{~cm}^{3} \mathrm{~mol}^{-1}\end{array}$ & $\begin{array}{c}\mathrm{Sv} \mathrm{cm} \\
\mathrm{L}^{1 / 2} \mathrm{~mol}^{-3 / 2}\end{array}$ & $\begin{array}{c}\Phi_{\mathrm{v}}{ }^{0} \\
\mathrm{~cm}^{3} \mathrm{~mol}^{-1}\end{array}$ & $\begin{array}{c}\mathrm{Sv} \mathrm{cm} \\
\mathrm{L}^{1 / 2} \mathrm{~mol}^{-3 / 2}\end{array}$ \\
\hline & \multicolumn{2}{|c|}{0 wt. $\% i-\mathrm{PrOH}$} & \multicolumn{2}{|c|}{10 wt. $\% i$-PrOH } & \multicolumn{2}{|c|}{20 wt. $\% i$-PrOH } \\
\hline 25 & 60.50 & 1.88 & 55.00 & 38.15 & 40.00 & 40.15 \\
\hline 30 & 60.66 & 1.92 & 56.25 & 38.46 & 40.50 & 32.00 \\
\hline \multirow[t]{2}{*}{35} & 62.67 & 1.98 & 59.00 & 29.41 & 42.05 & 31.65 \\
\hline & \multicolumn{2}{|c|}{30 wt. $\%$ i-PrOH } & \multicolumn{2}{|c|}{40 wt. $\%$ i-PrOH } & \multicolumn{2}{|c|}{50 wt. $\%$ i-PrOH } \\
\hline 25 & 33.00 & 52.63 & 37.00 & 58.00 & 50.00 & 12.22 \\
\hline 30 & 36.00 & 48.00 & 38.01 & 6.60 & 60.00 & 10.60 \\
\hline \multirow[t]{2}{*}{35} & 46.50 & 32.70 & 39.05 & 18.85 & 66.00 & 5.00 \\
\hline & \multicolumn{2}{|c|}{60 wt. $\%$ i-PrOH } & \multicolumn{2}{|c|}{70 wt. $\%$ i-PrOH } & \multicolumn{2}{|c|}{80 wt. $\%$ i-PrOH } \\
\hline 25 & 8.00 & 12.33 & 6.50 & 12.50 & 58.00 & 36.00 \\
\hline 30 & 43.00 & 15.00 & 44.75 & 25.00 & 58.09 & 18.27 \\
\hline \multirow[t]{2}{*}{35} & 50.00 & 29.50 & 54.00 & 15.00 & 59.00 & 10.33 \\
\hline & \multicolumn{2}{|c|}{90 wt.\% i-PrOH } & & & & \\
\hline 25 & 59.00 & 46.43 & & & & \\
\hline 30 & 60.75 & 11.54 & & & & \\
\hline 35 & 63.50 & 5.67 & & & & \\
\hline
\end{tabular}


Where $\mathrm{V}_{\mathrm{vw}}$ is the intrinsic or the Vander Wall's volume, $\mathrm{V}_{\mathrm{f}}$ the void volume, $\mathrm{V}_{\mathrm{s},}$, the contribution from solute-solute-solvent interactions and $\mathrm{V}_{\mathrm{h}}$ that from hydrophobic hydration taking $\left(\mathrm{V}_{\mathrm{vw}}+\mathrm{V}_{\mathrm{f}}\right)$ to be the identical in aqueous isopropanol and in water, the expected changes $\left(\mathrm{V}_{\mathrm{s}}+\mathrm{V}_{\mathrm{h}}\right)$ should explain the observed trains in $\Phi_{\mathrm{v}}{ }^{0}$. The $\left(\mathrm{V}_{\mathrm{s}}+\mathrm{V}_{\mathrm{h}}\right)$ for MCAA in water-isopropanol can be given as;

$$
\left(\mathrm{V}_{\mathrm{s}}+\mathrm{V}_{\mathrm{h}}\right) \text { in water isopropanol }=\mathrm{Vss}-\mathrm{Vsw}+\mathrm{V}_{i \mathrm{PrOH} i \mathrm{PrOH}}-\mathrm{Vs}_{\mathrm{iPrOH}}+\mathrm{VWW}
$$

Where $\mathrm{s}$ stands for solute MCAA, $\mathrm{w}$ for water, $i$-PrOH for isopropanol. Enlarge in $\Phi_{\mathrm{v}}{ }^{0}$ with raise of temperature can be recognized to decline in $-\mathrm{Vsw}$ and $-\mathrm{V}_{\mathrm{iPrOH}}$ due to preferential salt - salt (Vss) interactions. Input from changes in $\mathrm{VWw}_{\mathrm{w}}$ and $\mathrm{V}_{\mathrm{iPrOH}} \mathrm{iPrOH}$ can be taken as comparatively small ${ }^{8}$.

The option of positive Sv value is accounted for strong electrostatic ion-ion interactions due to bulky size of acetate ion which end result in interionic penetration ${ }^{9}$ foremost to ion ion interaction. The applicability of John`s - Dole equation is discovered by linear plot.

$\eta \mathrm{r}-1 / \sqrt{C} v s . \sqrt{C}$ at all temperatures the values of constant $\mathrm{A}$ and $\mathrm{B}$ precise by the intercept and slope of the plots respectively are given in Table 2. "A' values are minute but positive in solutions in $10,20,60,70,80$ and 90 wt.\% isopropanol representing in significant solute-solute interactions. However unexpected negative values of "A" in MCAA solution in 30, 40, 50 and 100 wt.\% isopropanol can not give details Falkenhagen theory. This uncharacteristic performance of MCAA in these solution as regards the symbol of ' $A$ ' is comparable to that obtained for MCAA in additional mixed solvents ${ }^{10,11}$.

Table 2. A and B Parameters of Jone`s-Dole equation at different compositions and diffrent temperatures

\begin{tabular}{crrrrrr}
\hline $\begin{array}{c}\text { Wt.\% } \\
i \text {-PrOH }\end{array}$ & \multicolumn{1}{c}{$\mathrm{A}$} & \multicolumn{1}{c}{$\mathrm{B}$} & \multicolumn{1}{c}{$\mathrm{A}$} & \multicolumn{1}{c}{$\mathrm{B}$} & $\mathrm{A}$ & \multicolumn{1}{c}{$\mathrm{B}$} \\
\hline \multicolumn{7}{c}{$25^{\circ} \mathrm{C}$} \\
\hline 0 & 0.026 & 0.031 & 0.040 & -0.050 & 0.056 & -0.760 \\
20 & 0.029 & -0.032 & 0.041 & -0.052 & 0.057 & -0.790 \\
30 & -0.142 & 0.136 & -0.112 & 0.100 & -0.130 & 0.025 \\
40 & -0.150 & 0.087 & -0.121 & 0.105 & -0.122 & 0.027 \\
50 & -0.0044 & 0.038 & -0.047 & 0.037 & -0.048 & 0.035 \\
60 & 0.052 & -0.059 & -0.051 & 0.039 & -0.041 & 0.038 \\
70 & 0.057 & -0.061 & 0.065 & -0.064 & 0.059 & -0.052 \\
80 & 0.013 & -0.010 & 0.015 & -0.007 & 0.014 & -0.001 \\
90 & 0.014 & -0.013 & 0.013 & -0.009 & 0.015 & -0.003 \\
100 & -0.014 & -0.030 & -0.030 & -0.038 & -0.017 & -0.020 \\
\hline
\end{tabular}

The positive 'B' and negative $\frac{d B}{d T}$ values for MCAA in solution in 30, 40 and $50 \mathrm{wt} . \%$ isoprpanol indicate strong solute- solvent interaction making MCAA as structure promoter ${ }^{12}$ in these solvent mixtures however negative ' $\mathrm{B}$ ' and positive $\frac{d B}{d T}$ values for MCAA in solution 10, 20,60, 70, 80 and 100 wt.\% isopropanol suggest that MCAA behaves as structure breaker in these solvent mixtures ${ }^{12}$. The variation of sound velocity with concentration $(\mathrm{C})$ of solute is given by

$$
\frac{d U}{d C}=-\frac{U}{2}\left[\frac{1}{\beta a d} x \frac{d \beta a d}{d c}+\frac{d \beta a d}{d c}+\frac{1}{\rho} x \frac{d \rho}{d C}\right]
$$


Magnitudes of positive values of $\left[\frac{1}{\rho} x \frac{d \rho}{d c}\right]$ are smaller than negative magnitude of $\left[\frac{1}{\beta a d} x \frac{d \beta a d}{d c}\right]$ making $\frac{d U}{d C}$ positive in solution in $10,20,30,40$ and $100 \mathrm{wt} . \%$ isopropanol. However in solution in 50,60, 70, 80 and 90 wt.\% isopropanol $\frac{1}{\rho} x \frac{d \rho}{d C}$ as well as $\frac{1}{\beta a d} x \frac{d \beta a d}{d C}$ are positive making $\frac{d U}{d C}$ negative. Thus the fact that increase of velocity (U), decrease of adiabatic compressibility, decrease of inter molecular free length $\left(\mathrm{L}_{\mathrm{f}}\right)$, increase of specific acoustic impendence $(Z)$ and decrease of salvation numbers $\left(S_{n}\right)$ with increase of molar concentration of MCAA in 10, 20, 30, 40 and $100 \mathrm{wt} \%$ isopropanol at all temperature is indicative of the increase of intermolecular forces with the addition of MCAA forming aggregates of solvent molecules around solute ions ${ }^{13}$ supports the strong solute-solvent interactions due to which structural arrangement is affected ${ }^{14}$ decrease of velocity $(U)$, increase of adiabatic compressibility, increase of intermolecular free length $\left(\mathrm{L}_{\mathrm{f}}\right)$, decrease of specific acoustic impendence $(\mathrm{Z})$ and increase of salvation number $\left(\mathrm{S}_{\mathrm{n}}\right)$ with increase of MCAA concentration in 50, 60. 70.80 and $90 \mathrm{wt} . \%$ isopropanol at all temperatures supports the existence of solute-solute interactions in these solutions. Similar behavior is observed at other temperature.

Apparent molar compressibility value $\left(\Phi_{\mathrm{k}}\right)$ vary linearly with $\sqrt{C}$ all temperatures obeying Gucker's limiting law.

$$
\Phi_{\mathrm{k}}=\Phi_{\mathrm{k}}{ }^{0}+\mathbf{S}_{\mathrm{k}} \sqrt{C}
$$

The intercept $\left(\Phi_{\mathrm{k}}{ }^{0}\right)$ and slopes $\left(\mathrm{S}_{\mathrm{k}}\right)$ of linear plots of $\Phi_{\mathrm{k}}$ versus $\sqrt{C}$ are listed in Table 3 . The negative $\Phi_{\mathrm{k}}{ }^{0}$ and positive $S_{\mathrm{k}}$ in solution in 10, 20, 30, 40 and $100 \mathrm{wt} . \%$ isopropanol indicates strong electrostatic solute-solvent interaction, while the positive. $\Phi_{\mathrm{k}}{ }^{0}$ and negative $\mathrm{S}_{\mathrm{k}}$ values in solution in 50,60, 70, 80 and $90 \mathrm{wt} \%$ isopropanol suggest that solvent molecules are loosely attached to solute. The adiabatic compressibility of MCAA solution are found to obey Bachem's relation.

$$
\beta_{a d}=\beta_{a d}^{0}+A C+B C^{\frac{3}{2}}
$$

$\beta_{a d}, \beta_{a d}^{0}$ and $\mathrm{C}$ have their usual meanings ${ }^{15}$. Constant ' $\mathrm{A}$ ' and ' $\mathrm{B}$ ' are intercepts and slopes of linear plots of $\beta_{a d}-\beta_{a d}^{0}$ versus $\sqrt{C}$ are included in Table 3.

Table 3. $\Phi_{\mathrm{k}}{ }^{0}, \mathrm{~S}_{\mathrm{k}}, \mathrm{A}$ and B parameters for MCAA in different compositions and different temperatures

\begin{tabular}{crrrr}
\hline Wt.\% & i-PrOH & \multicolumn{5}{c}{$\mathrm{A}^{1} 10^{12}$} & \multicolumn{1}{c}{$\mathrm{B}^{12} 10^{12}$} & $\Phi_{\mathrm{k}}{ }^{0} \times 10^{9}$ & $\mathrm{~S}_{\mathrm{k}} \times 10^{9}$ \\
\hline 10 & -17.4 & 0.33 & -13.0 & 0.04 \\
20 & -11.45 & 0.25 & -10.8 & 0.08 \\
30 & 5.5 & 0.16 & -3.7 & 0.12 \\
40 & 0.1 & 0.8 & -0.09 & -0.36 \\
50 & 4.5 & -0.20 & 6.6 & -0.40 \\
60 & 8.5 & -0.15 & 9.6 & -0.54 \\
70 & 11.8 & -0.10 & 15.7 & -0.08 \\
80 & 10.6 & -0.008 & 16.09 & -0.06 \\
90 & 12.5 & -0.06 & 16.4 & -0.1 \\
\hline
\end{tabular}




\begin{tabular}{|c|c|c|c|c|}
\hline 100 & 15.1 & 0.01 & -8.9 & 8.80 \\
\hline \multicolumn{5}{|c|}{$30^{\circ} \mathrm{C}$} \\
\hline 10 & -16.1 & 0.12 & -11.5 & 0.05 \\
\hline 20 & -10.4 & 0.118 & -9.3 & 0.07 \\
\hline 30 & -6.3 & 0.11 & -5.1 & 0.11 \\
\hline 40 & -3.2 & 0.09 & -4.6 & 0.13 \\
\hline 50 & 11.7 & -0.07 & 15.7 & -0.68 \\
\hline 60 & 10.6 & 0.06 & 14.3 & -0.078 \\
\hline 70 & 9.8 & -0.09 & 11.5 & -0.11 \\
\hline 80 & 6.8 & -0.05 & 10.2 & -0.12 \\
\hline 90 & 5.9 & -0.06 & 11.1 & -0.14 \\
\hline 100 & -20.1 & -0.03 & -12.7 & 17.86 \\
\hline \multicolumn{5}{|c|}{$35^{\circ} \mathrm{C}$} \\
\hline 10 & -15.3 & 0.50 & -8.5 & 0.04 \\
\hline 20 & -10.4 & 0.40 & -5.4 & 0.1 \\
\hline 30 & -7.7 & 0.1 & -3.2 & 0.15 \\
\hline 40 & -5.3 & 0.07 & 18.2 & 0.16 \\
\hline 50 & 14.0 & -0.06 & 16.8 & -.007 \\
\hline 60 & 15.2 & -.003 & 18.2 & -0.06 \\
\hline 70 & 17.3 & -.004 & 19.1 & -0.52 \\
\hline 80 & 8.5 & -.007 & 15.6 & -0.08 \\
\hline 90 & 7.5 & -.005 & 13.1 & -0.09 \\
\hline 100 & -6.5 & 0.2 & -10.2 & 18.00 \\
\hline
\end{tabular}

\section{Acknowledgement}

The author expresses gratitude to Mr. Raosaheb Patil for the support and encouragement

\section{References}

1. Barthel J, Gores H J, Schmeer G and Watcher R, Nonaqueous electrolyte Solutions in Chemistry and Modern Techonology, Springer Verlag, Heidelberg, Germasny, 1983.

2. Chantooni M K, (Jr.) and Kolthoff M I, J Phys Chem., 1978, 82(9), 994-1000; DOI:10.1021/j100498a006

3. Ito N, Fujiyama T and Udagawa V, Bull Chem Soc., (Japan), 1981, 54, 2573-2578; DOI:10.1246/bcsj.54.2573

4. Nikam P S and Mehandi Hasan, J Chem Eng Data, 1988, 30(2), 165-169; DOI:10.1021/je00052a032

5. Harned H S and Owen B B, The Physical Chemistry of Electrolyte Solutions, Reinhold, New York, 1958, 161.

6. Mishra A K and Ahiuwalia J C, J Phys Chem., 1984, 88(1), 86; DOI:10.1021/j150645a021

7. Wadi R K and Gopal R K, J Chem Eng Data, 1992, 37(4), 377-386; DOI:10.1021/je00008a002

8. Nemetny G and Scherage H A, J Chem Phys., 1962, 36, 3382; DOI:10.1063/1.1732472

9. Gopal R, Agrawal D K and Kumar R, Z Phys Chem., 1973, 84, 141.

10. Nikam P S and Mehandi Hasan, Curr Sci (India), 1984, 53, 260.

11. Nikam P S and Hirey A R, Indian J Pure Appl Phys., 1988, 24, 502. 
12. Sharma T S and Ahluwalia J C, Rev Chem Soc., 1973, 2, 217.

13. Prakash S, Ichihaporia F M and Pandey J D, J Phys Chem., 1964, 58, 3078.

14. Eyring H and Kincaid J K, J Chem Phys., 1932, 6, 620; DOI:10.1063/1.1750134

15. Nikam P S and Hirey A R, Indian J Pure Appl Phys., 1992, 29, 601. 